\author{
Laurent Kodjikian \\ Justus G. Garweg \\ Jacques Fleury \\ François Rocher \\ Carole Burillon \\ Jean-Daniel Grange
}

\section{Bilateral rhegmatogenous retinal detachment after external-beam radiotherapy: just a coincidence?}

Received: 17 September 2003

Revised: 9 December 2003

Accepted: 17 December 2003

Published online: 24 March 2004

(C) Springer-Verlag 2004

This study received no funding, and the authors had no financial or proprietary interest in it

Laurent Kodjikian had full access to all available data and takes full responsibility for their integrity and for the accuracy of the analysis

Study previously presented at the meeting of the French Ophthalmologic Society on 10 May 2003 in Paris, France

L. Kodjikian ( J ) Fleury · J.-D. Grange Department of Ophthalmology,

Croix-Rousse Hospital,

103, grande rue de la Croix-Rousse, 69004

Lyon, France

e-mail: kodjikian.laurent@wanadoo.fr

Tel.: +33-4-72071718

Fax: +33-4-72071708

L. Kodjikian · J. G. Garweg

Department of Ophthalmology, Inselspital, University of Bern,

Bern, Switzerland

L. Kodjikian · C. Burillon Department of Ophthalmology,

Ed. Herriot Hospital,

Lyon, France

F. Rocher

Department of Radiotherapy du Parc,

Clinique Sainte-Marie,

Chalon-sur-Saône, France

\begin{abstract}
Purpose: To report an unusual case of almost simultaneous bilateral rhegmatogenous retinal detachment in the context of externalbeam radiotherapy for a tumor at a non-ocular target site and in the absence of pre-existing ocular pathology. Methods: Observational case report with review of corresponding literature. Results: A 63-year-old man was referred for bilateral retinal detachment which was associated with many horseshoe tears and proliferative vitreoretinopathy. He had undergone surgery for a carcinoma of the left maxillary sinus 4 months prior to the presentation and had then received external-beam radiotherapy for 3 months. There was no familial history of retinal detachment and/or eye trauma in this hyperopic patient with clear native lenses. No chorioretinal pathology was apparent that could have predisposed the retinas to tearing. Conclusions: Simultaneous bilateral retinal detachment is exceptional, especially in a patient with no risk factors. The effect of radiotherapy on the vitreoretinal interface is discussed in the light of existing data and may have been responsible for our patient's retinal detachment.
\end{abstract}




\section{Introduction}

Rhegmatogenous retinal detachment (RD) is not an acknowledged complication of ocular radiation, although such cases have been reported $[9,11]$. Indeed, the possibility of a correlation has not been systematically addressed. Following the few published cases, we present here the first report of bilateral rhegmatogenous RD with proliferative vitreoretinopathy (PVR) after external-beam radiotherapy for a tumor at a non-ocular site, in this case a carcinoma of the left maxillary sinus. The clinical features of this remarkable case are described and the possible pathological mechanisms underlying it are discussed.

\section{Case report}

A 63-year-old man was referred to our hospital for bilateral rhegmatogenous RD on 27 August 2002. He had noted the existence of floaters and a deterioration in visual acuity, first of the left eye ( 8 weeks prior to presentation) and then of the right eye ( 2 weeks prior to presentation). He had undergone surgery for a carcinoma of the left maxillary sinus in May 2002 and had then received externalbeam radiotherapy from 19 June 2002 until 5 August 2002. He was administered a total photon (X-ray) dose of 66 Gy. Despite the utmost care to minimize ocular exposure to radiation, this nevertheless had occurred to a significant degree. The left vitreoretinal interface had incurred a dose of 36-45 Gy, the right, 10-15 Gy (Fig. 1).

There was no familial history of RD or eye trauma in this hyperopic patient with clear native lenses. Examination of the right fundus revealed the presence of a macula-sparing RD involving the superior half of the retina, with five horseshoe tears and PVR

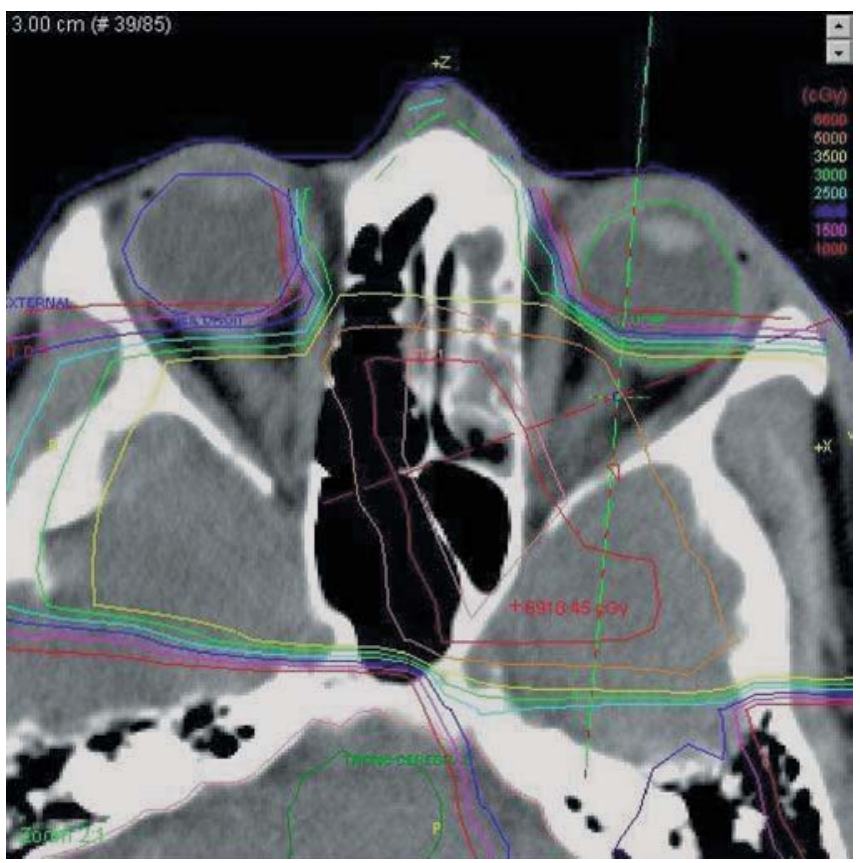

Fig. 1 Isodose curves on a computed tomographic scan, revealing the irradiation field for each eye

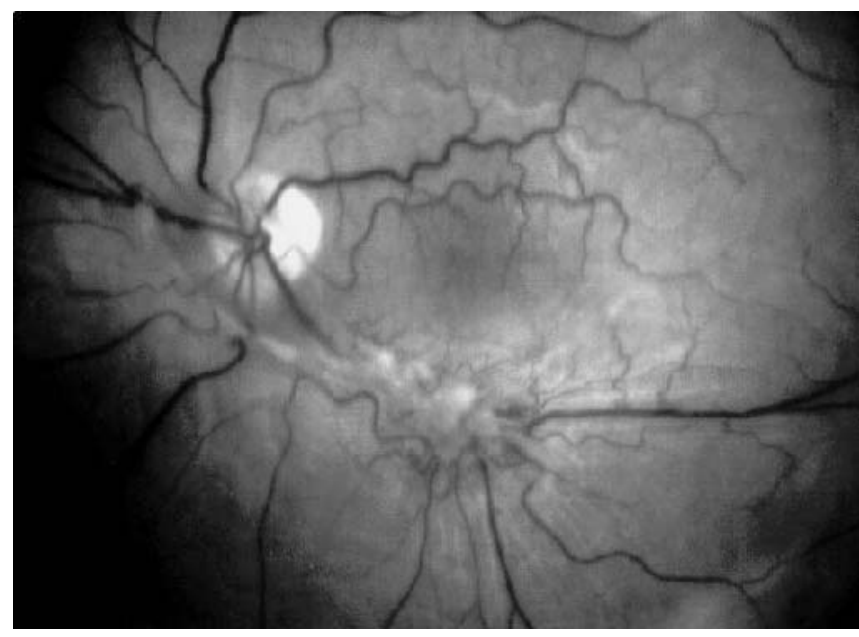

Fig. 2 Fundus photograph of the left eye 1 month after surgery, illustrating the reattached retina with a star fold (due to proliferative vitreoretinopathy) close to the temporal inferior vessels

(grade B). In the left eye, the RD only just spared the macula; it was characterized by four horseshoe tears and PVR (grade B). No chorioretinal pathology was apparent that could have predisposed the retinas to tearing. Posterior detachment of the vitreous was complete in both eyes, except in the meridian of three retinal tears (in each eye), where it adhered to the anterior flap of the tear in the absence of scleral pressure. Clusters of pigmented cells were apparent in the vitreous of both eyes ("tobacco dust"). Examination of the fundus 1 year prior to external-beam radiotherapy had revealed no pathological findings. Standard ultrasonography performed in August 2002 disclosed no chorioretinal tumor. The carcinoma of the sinus was clinically quiescent and was deemed to have been successfully treated. A work-up for metastases yielded negative results. Ocular surgery was successfully performed first on the right eye (buckling surgery using cerclage) and 2 weeks later on the left eye (vitrectomy and cerclage), by which time the PVR had increased from grade B to grade C1 (Fig. 2). Unfortunately the vitrectomy material was not submitted to cytological examination.

\section{Discussion}

This case is of particular in interest in being the first documented instance of bilateral rhegmatogenous RD development after external-beam radiotherapy for a tumor at a non-ocular target site and in the absence of pre-existing ocular pathology.

In the context of external-beam radiotherapy, serous RD has been observed as a complication either of metastasis or of the therapy itself of intraocular malignancies $[2,9,10]$, but has never been reported for extraocular malignancies without any ocular involvement. However, in our case, clinical examination and ultrasonography excluded the presence of a chorioretinal tumor. Furthermore, numerous retinal tears were observed, the surgical repair of which resulted in prompt and complete bilateral retinal reattachment. This latter circumstance supports the diagnosis of rhegmatogenous RD. 
$\mathrm{RD}$ arising from retinal breaks occurs in about 12 per 100,000 persons annually, and in half as many non-traumatic phakic patients [8]. Bilateral RD has been estimated to occur in 2 per 1,000,000 phakic persons per year [8]. In most of the articles dealing with bilateral RD, however, the authors do not distinguish between simultaneous and delayed development in the second eye. In our experience, the incidence of simultaneous bilateral RD is certainly much lower, and probably highly exceptional. In one study, the incidence of bilateral RD is reported to be $13 \%$ and that of simultaneous bilateral RD only $1.4 \%$ [4]. In another report, only one patient $(0.4 \%)$ at a particular retina centre was diagnosed with simultaneous bilateral RD over a period spanning 8.5 years [3]. That patient's high myopia (12 D) was believed to be the disposing factor. Indeed, such risk factors are regularly reported for patients with bilateral RD, and include also chorioretinal lesions (such as lattice degeneration or focal pigmentary proliferation), cataract surgery, advanced age, familial antecedents of RD and/or eye trauma $[3,5]$. Our patient, however, fell into none of these categories. Moreover, the RD was highly exceptional in that occurred almost simultaneously in both eyes, first in the eye that had received the higher dose of radiation. In this eye, PVR was also more severe. Hence, the bilateral rhegmatogenous $\mathrm{RD}$ in this specific case may be considered as an ocular side-effect of extraocular radiotherapy, not merely a coincidence.

Little has been reported about the effects of radiotherapy on the vitreous and the vitreoretinal interface. Radiation could conceivably have induced contraction and detachment of the vitreous. Clinically, posterior detachment of the vitreous was virtually complete in both of our patient's eyes at the time of surgery, with vitreal traction being evident on some of the tears. Theoretically, radiotherapy could also have altered the vitreoretinal interface, inducing or reinforcing adhesions between the posterior vitreal cortex and the internal limiting membrane of the retina, and thereby enhancing the risk of retinal tearing [2]. Remarkably, abnormal deposits of fibrin have been encountered in histopathological sections of 33 eyes that had undergone radiotherapy for retinoblastomas [6]. These occurred not only intra- but also extra-vascularly, within the retinal stroma, and they could conceivably have induced strong vitreoretinal adhesion. No correlation was found to exist between the severity of fibrin proliferation and the radiation dose delivered or the age of the patient. Moreover, there existed no corresponding retinal changes in the control non-irradiated eyes, which confirmed that the deposits of fibrin were related not to the underlying disease but to the radiotherapy. Such fibrin deposits were not visible biomicroscopically in our patient. In another histopathological report dealing with the morphological changes induced by fractionated external-beam radiotherapy in the eyes of minipigs, a homogeneously distributed layer of collagen fibrils appeared on the vitreal aspect of the inner limiting membrane and was believed to have enhanced the adhesion of the interface to the retina [1]. Radiation is also known to have a direct impact on retinal pigmented epithelial (RPE) cells, animal experiments having revealed a dose-dependent effect on their morphology and proliferation [1]. These morphological changes might impair the adhesion of epithelial cells to the sensory retina and Bruch's membrane. In our patient, such changes could have facilitated the induction of RD by retinal tearing, which in turn resulted from vitreal contraction. In the histopathological study reported by Alberti and El-Hifnawi, local hypertrophy of the RPE cells was also observed in some eyes [1]. In our patient, this phenomenon may have accounted for the abundance of pigmented cells within the vitreous of both eyes and may have contributed to an amplified proliferative response of these cells and to the rapid evolution of the PVR [1]. Indeed, RPE cells have been recognized to be a major component of contractile cellular membranes in PVR [7]. They may be responsible for induction of PVR, since intravitreal injection of RPE cells in rabbits results in cellular membrane formation and traction RD similar to that seen in PVR [13]. One could argue that radiotherapy is known to suppress rather than exacerbate PVR in the experimental setting [12]. In experiments with rabbits, ocular irradiation after the injection of fibroblasts or RPE cells into the vitreous did indeed suppress PVR and tractional $\mathrm{RD}$, but radiotherapy performed before cell implantation was not effective in this capacity [16]. Furthermore, radiotherapy does not suppress traumatic PVR [15]. Clearly, several parameters may influence the effect of radiotherapy on vitreal and retinal structures, but our patient was obviously the victim of radiation-induced vitreoretinal traction, with consequent rhegmatogenous RD and PVR.

It would of course be wrong to assume that a temporal relationship between two events, necessarily implies a causal link. However, several arguments favor a non-coincidental nature of the relationship between radiotherapy and the development of bilateral rhegmatogenous RD in our patient, including the almost simultaneous occurrence of the disease in both eyes and the pathophysiological evidence for the deleterious effects of radiation on the vitreous, the vitreoretinal interface and retinal cells. One could argue that if radiation was indeed the culprit, then thousands of patients who have received radiotherapy for uveal melanomas should have developed rhegmatogenous RD. Excluding articles that deal with the simultaneous diagnosis of choroidal melanomas and $\mathrm{RD}$, we unearthed no documented evidence of this complication of radiation in the literature, with but two exceptions. The Harvard Cyclotron Team in Boston reported six cases of rhegmatogenous RD development after either proton-beam irradiation (five patients) or ${ }^{125}$ I plaque radiotherapy (one patient) [9]. The Wills Eye Hospital in Philadelphia reported on 270 patients who had been treated for choroidal melanomas by combined plaque radiotherapy and 
transpupillary thermotherapy and furnished a more precise estimation of the occurrence of this complication [14]. Kaplan-Meier estimates of the rate of rhegmatogenous RD were $2 \%$ at 2 and 5 years. This rate is not negligible and argues in favor of our assumption. Hitherto, the rate of rhegmatogenous RD following radiotherapy may have been underestimated because physicians were not aware of the problem. Indeed, recently a suspected relationship between rhegmatogenous RD and radiotherapy for retinoblastomas has been reported [11]. After careful consideration of all the above-mentioned literature, there appears to be histopathological evidence from retinoblastoma and animal models, and epidemiological evidence from uveal melanoma, that rhegmatog- enous RD is a result not of the initial pathology but of the radiotherapy. To the best of our knowledge, our patient represents the first documented case of bilateral rhegmatogenous RD development after irradiation of a nonocular target site.

We finally assume that our patient must have been exceptionally sensitive to radiation, since no similar case has been reported hitherto. Although a single case report does not carry much weight, we would like to stress that a relationship may well exist between ocular irradiation and rhegmatogenous RD. We encourage physicians to observe their patients closely for symptoms of the disease in this context.

\section{References}

1. Alberti WE, El-Hifnawi ES (1993) Morphologic changes after fractionated external-beam radiotherapy of minipig eyes. In: Alberti W, Sagerman R, eds. Radiotherapy of intraocular tumors. Springer, Berlin Heidelberg New York, pp 400-414

2. Bovey EH, Fernandez-Ragaz A, Héon E, Balmer A, Munier FL (1999) Rhegmatogenous retinal detachment after treatment of retinoblastoma. Ophthalmic Genet 20:141-151

3. De Rötth A (1939) Bilateral detachment of the retina. A heredodegenerative disease. Arch Ophthalmol 22:809831

4. Delaney WV Jr, Oates RP (1978) Retinal detachment in the second eye. Arch Ophthalmol 96:629-634

5. Dumas J (1966) Chorioretinal lesion predisposing to retinal breaks. Am J Ophthalmol 61:620-630

6. Egbert PR, Fajardo LF, Donaldson SS, Moazed K (1980) Posterior ocular abnormalities after irradiation for retinoblastoma: a histopathological study. Br J Ophthalmol 64:660-665
7. Glaser BM, Vidaurri_Leal J, Michels RG, Campochiaro PA (1993) Cryotherapy during surgery for giant retinal tears and intravitreal dispersion of viable retinal pigment epithelial cells. Ophthalmology 100:466-470

8. Haimann MH, Burton TC, Brown CK (1982) Epidemiology of retinal detachment. Arch Ophthalmol 100:289292

9. Haimovici R, Mukai S, Schachat AP, Haynie GD, Thomas MA, Meredith TA, Gragoudas ES (1996) Rhegmatogenous retinal detachment in eyes with uveal melanoma. Retina 16:488496

10. Inoue $\mathrm{K}$, Numaga J, Kaji Y, Toda J, Kato S, Sakurai M, Ikeda M, Motoi N, Murakami T, Fujino Y (2000) Bilateral choroidal metastases secondary to uterocervical carcinoma of the squamous cell type. Am J Ophthalmol 130:682-684

11. Lim TH, Robertson DM (2000) Presumed rhegmatogenous retinal detachment in patients with retinoblastoma. Retina 20:22-27
12. Palan P, Tan KEWP (1973) Radiotherapy in the management of retinal detachment complicated by traction processes. Radiol Clin Biol 42:191198

13. Radtke ND, Tano Y, Chandler D, Machemer R (1981) Simulation of massive periretinal proliferation by autotransplantation of retinal pigment epithelial cells in rabbits. Am J Ophthalmol 91:76-87

14. Shields CL, Cater J, Shields JA, Chao A, Krema H, Materin M, Brady LW (2002) Combined plaque radiotherapy and transpupillary thermotherapy for choroidal melanoma. Tumor control and treatment complications in 270 consecutive patients. Arch Ophthalmol 120:933-940

15. Velikay M, Binder S, Stolba U, Wedrich A, Datlinger P, Handl-Zeller L (1994) Experimental perforating injury with high energy electrons to inhibit intraocular proliferation. Acta Ophthalmol Scand 72:337-340

16. Velikay M, Stolba U, Wedrich A, Datlinger P, Akramian J, Binder S (1994) The antiproliferative effect of fractionized radiation therapy: optimization of dosage. Doc Ophthalmol $87: 265-269$ 